\title{
The Year of the Filipino Pathologist
}

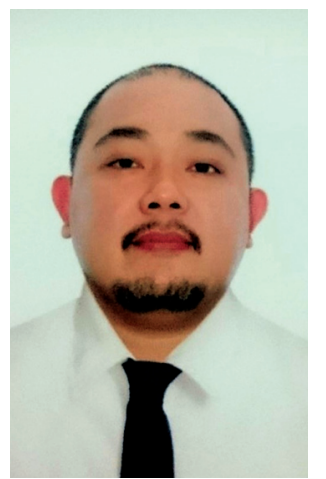

Sitting here in the quarantine facility, waiting for my rTPCR result, after getting exposed to a positive colleague, I now get a sort of breather: a reprieve from the frenzied activities related to the COVID-19 laboratory response over the last six months.

Outside, everything looks the same but feels different at the same time. Limited outside activities, social distancing, mass wearing of masks have become the new normal. Strict curfews, relatively less traffic, closed stores and deserted streets, now describe our once-busy evenings. Work itself has adapted through virtual meeting places and shared online documents. The pandemic has redefined and continues to redefine life as we all know it in this generation.

At this point, our Institute has tested more than 150,000 specimens, the largest number of specimens our agency has tested for a single public health threat. Thousands of results have been generated and transmitted to the Department of Health as part of the laboratory's contribution to the response. But one molecular laboratory cannot do it alone. After several thousands of miles and hours of travel time, our technical team has assessed, trained, provided technical assistance to, and activated over 90 molecular laboratories proficient for SARS-COV-2 testing, giving rise to a living, breathing laboratory network. We now have a daily capacity greater than 20,000 specimens compared to 300 when we started in February.

Outside, the Philippine Society of Pathologists has become more active in voicing out its collective stand on COVID-19 laboratory issues, issuing a position paper on strategies to improve the accessibility and scope of testing. In the last two months, we noted the increasing representation of pathologists in various tri-media platforms, talking and being consulted about COVID-19 laboratory testing. The Society has also supported a group of pathologist-researchers in incubating, finalizing, and implementing a pragmatic, operations research on pooled testing, findings of which we hope to soon read in our journal.
Virtual technical working groups have been established to discuss antibody-based testing, instrument-based serologic assays, closed molecular systems, and other issues. Enabled by technology, the pandemic has catalyzed more technical, managementrelated, and quality assurance discussion among heads of laboratories.

With laboratory testing considered as the central basis for contact tracing and treatment strategies, and the increased recognition of the role of the laboratory in public health, 2020 is turning out to be the year of the Filipino pathologist. We can only hope that the momentum is sustained, and pathologists continue to assert their expertise and provide leadership and direction in concerns related to the laboratory.

Like PCR testing which cannot simply be done by a single laboratory entity, understanding our enemy cannot be accomplished by one agency alone. We are all together in fighting this submicroscopic entity and a lot still remains to be fathomed about the pathology it causes. Research should not cease because of the pandemic.

I am issuing this call to all to all Filipino pathologists: I enjoin you to contribute to the global knowledge base on COVID-19. Publish your researches, case reports, interesting images and findings, related to the pandemic in our journal. Engage with your clinical colleagues in their studies on the management of this disease. Share your assay validations of PCR and serologic test kits, operational improvements, biosafetybiosecurity enhancements and best practices in your laboratories. Let us learn from each other and help each other beat this disease.

The PJP is one with the Society in the fight against SARS-CoV2. We are here. At your service.

\section{Amado O. Tandoc III, MD, FPSP}

Editor-in-Chief

https://doi.org/10.21141/PJP.2020.01 